\title{
Kinetic Alfvén wave generation by velocity shear in collisionless plasmas
}

\author{
T. Maiorano ${ }^{1}$, A. Settino ${ }^{1}$, F. Malara ${ }^{1} \dagger$, O. Pezzi ${ }^{2,3}$, F. Pucci ${ }^{4}$, and F. \\ Valentini $^{1}$ \\ ${ }^{1}$ Dipartimento di Fisica, Università della Calabria, 87036 Rende (CS), Italy \\ ${ }^{2}$ Gran Sasso Science Institute, Viale F. Crispi 7, I-67100 L. Aquila, Italy \\ ${ }^{3}$ INFN/Laboratori Nazionali del Gran Sasso, Via G. Acitelli 22, I-67100 Assergi (AQ), Italy \\ ${ }^{4}$ Centre for Mathematical Plasma Astrophysics, Department of Mathematics, KU Leuven, \\ Celestijnenlaan 200B, 3001 Leuven, Belgium
}

(Received xx; revised xx; accepted $\mathrm{xx}$ )

The evolution of a linearly-polarized, long-wavelength Alfvén wave -propagating in a collisionless magnetized plasma with a sheared parallel-directed velocity flow- is here studied by means of two-dimensional hybrid Vlasov-Maxwell (HVM) simulations. The unperturbed sheared flow has been represented by an exact solution of the HVM set of equations (Malara et al., Phys. Rev. E 97, 053212), this avoiding spurious oscillations that would arise from the non-stationary initial state and inevitably affect the dynamics of the system. We have considered the evolution of both a small and a moderate amplitude Alfvén wave, in order to separate linear wave-shear flow couplings from kinetic effects, the latter being more relevant for larger wave amplitudes. The phase-mixing generated by the shear flow modifies the initial perturbation, leading to the formation of small-scale transverse fluctuations at scales comparable with the proton inertial length. By analyzing both the polarization and group velocity of perturbations in the shear regions, we identify them as Kinetic Alfvén Waves (KAWs). In the moderate amplitude run, kinetic effects distort the proton distribution function in the shear region. This leads to the formation of a proton beam, at the Alfvén speed and parallel to the magnetic field. Such a feature, due to the parallel electric field associated with KAWs, positively compares with solarwind observations of suprathermal ions' populations, suggesting that it may be related to the presence of ion-scales KAW-like fluctuations.

\section{Introduction}

In several natural contexts low-frequency fluctuations display properties which are typical of an Alfvénic state: velocity and magnetic field perturbations are highly correlated (positively or negatively), while density and magnetic field intensity are affected by much less intense fluctuations. This situation is typically found in solar wind turbulence, mainly in low-latitude fast-speed streams, or in the high-latitude wind (e.g., Belcher \& Davis 1971; Bruno \& Carbone 2013). Recently, measures performed by the Parker Solar Probe spacecraft at small heliocentric distances have shown fluctuations in the solar wind emanating from an equatorial coronal hole with strong Alfvénic correlation, even in cases of very large amplitudes (the so-called switchbacks)(Bale et al. 2019). Another example is that of velocity fluctuations propagating along the magnetic field at the Alfvén speed detected in the solar corona (Tomczyk et al. 2007; Tomczyk \& McIntosh 2009), which have been interpreted as Alfvén waves.

$\dagger$ Email address for correspondence: francesco.malara@fis.unical.it 
In the framework of the magnetohydrodynamic (MHD) turbulence, such perturbations interact nonlinearly producing a cascade which moves fluctuating energy at increasingly smaller scales. In the presence of a background magnetic field $\mathbf{B}_{0}$, nonlinear interactions preferentially take place in the directions transverse to $\mathbf{B}_{0}$ (Shebalin et al. 1983 ; Carbone \& Veltri 1990; Oughton et al. 1994). Therefore, an anisotropy develops in the spectral space, with perpendicular wavevectors dominating over parallel ones at small scales. Indeed, solar-wind observations have shown the presence of a significant population of quasi-perpendicular wavevectors (Matthaeus et al. 1986, 1990; Carbone et al. 1995; Milano et al. 2001; Dasso et al. 2005; Matthaeus et al. 2012; Oughton et al. 2015). A further element, which can play a role in the turbulent dynamics, is given by the interaction of fluctuations with inhomogeneities associated with large scale structures, such as pressure-balanced structures or velocity shears (Ghosh et al. 1998). For instance, this happens in the solar wind around the heliospheric current sheet (Malara et al. 1996a) or at the interface between fast and slow speed streams (Roberts et al. 1991, 1992). Finally, fluctuations in the turbulent spectrum at scales of the order or lower than ion scales (ion inertial length and/or ion Larmor radius) are affected by dispersive and kinetic phenomena. In a collisionless plasma, these kinetic processes generate outof-equilibrium features in the particle distribution function: ion temperature anisotropy has been routinely observed in the solar wind (e.g., Hellinger et al. 2006); the proton distribution function in the solar wind and in the Earth's magnetosphere can include a beam directed in the direction parallel to the local magnetic field (Goodrich \& Lazarus 1976; Marsch et al. 1982; Marsch 2006; Sorriso-Valvo et al. 2019), with a drift speed of the order of the local Alfvén velocity (Goldstein et al. 2000; Tu et al. 2004). Physical processes which can lead to the formation of this beam have also been considered (Araneda et al. 2008; Valentini et al. 2008; Matteini et al. 2010; Valentini et al. 2011a, b; Narivuki et al. 2014a). More recently, a further level of velocity-space complexity, namely the presence of an enstrophy cascade towards smaller velocity-space scales, has been pointed out either in magnetosheath' observations (Servidio et al. 2017) and in numerical simulations (Pezzi et al. 2018; Cerri, Kunz \& Califano 2018), which are also useful to investigate the nature of cross-scale correlations between the inertial-range turbulent energy cascade and the small-scale kinetic processes in collisionless plasmas (Sorriso-Valvo et al. 2018).

A full description which takes into account the above features, namely, Alfvénic correlations, spectral anisotropy, the role of background inhomogeneities, and kinetic effects at ion scales, is a complex task. However, some insight can be gained by means of simplified approaches. In particular, in this paper we will focus on a specific aspect: the interaction between an Alfvénic perturbation and a bulk velocity inhomogeneity transverse to the background magnetic field, which produces small scales of the order of the ion inertial length in the transverse direction. This effect somehow mimics the anisotropic small-scale generation taking place in a turbulence, where large scales evolve on characteristic times which are larger than that of smaller scales.

Such a problem has been already analyzed in detail within the MHD description, where two distinct mechanisms are at work: phase-mixing, in which differences in phase speed produce a progressive de-phasing that bends wavefronts and increases the transverse component of the wavevector; and resonant absorption, in which the wave energy progressively concentrates in thin layers where a resonance condition is satisfied. These processes have been studied both by normal mode approaches (Kappraff \& Tataronis 1977; Mok \& Einaudi 1985; Steinolfson 1985; Davila 1987; Hollweg 1987; Califano et al. 1990, 1992) and by considering the evolution of an initial disturbance (Lee \& Roberts 1986; Malara et al. 1992, 1996b). Localized pulses (Kaghashvili 1999; Tsiklauri \& Nakariakov 
2002; Tsiklauri et al. 2003) have also been considered. The propagation of MHD waves in inhomogeneous magnetic fields containing null points has also been studied (Landi et al. 2005; McLaughlin et al. 2011; Pucci et al. 2015), finding a fast formation of small scales perpendicular to the ambient magnetic field. In 3D inhomogeneous equilibria this process has been considered in the small wavelength limit (Similon \& Sudan 1989; Petkaki et al. 1998; Malara et al. 2000), also within the problem of coronal heating (Malara et al. 2003, 2005, 2007).

When the wavelength of the perturbation has decreased until reaching values comparable with the ion inertial length, both dispersive and kinetic effects, which are neglected in the MHD approach, become important. In particular, an Alfvén wave with a wavevector which is (i) quasi-perpendicular to the background magnetic field, and (ii) of the order of the inverse of the ion inertial length, is generally indicated as "kinetic Alfvén wave" (KAW). KAWs have received considerable attention because observations have shown that the polarization of fluctuations at kinetic scale in the solar wind is consistent with the presence of KAW-like fluctuations (Chen et al. 2013). Since the MHD cascade favors nearly perpendicular wavevectors, the expectation within a wave perspective would be that fluctuations having a character resembling KAWs were naturally present at scales of the order of the ion inertial length. An extensive analysis of the KAW physics can be found in Hollweg (1999) (see also references therein for a more complete view on the subject). Many solar wind observational analyses (Bale et al. 2005; Sahraoui et al. 2009; Podesta \& TenBarge 2012; Salem et al. 2012; Chen et al. 2013; Kivani et al. 2013), theoretical works (Howes et al. 2008a; Schekochihin et al. 2009; Sahraoui et al. 2012) as well as numerical simulations (Gary \& Nishimura 2004; Howes et al. 2008b; Tenbarge \& Howes 2012) have suggested that fluctuations near the end of the MHD inertial cascade range may consist primarily of KAWs and that such fluctuations can play an important role in the dissipation of turbulent energy. Formation of KAWlike small-amplitude fluctuations has also been observed in numerical simulations of collisions between two counter-propagating Alfvénic wavepackets (Pezzi et al. 2017a, b. c) , a process which mimics nonlinear interactions among localized eddies in turbulence. Moreover, Landau damping and wave-particle resonant interactions can take place in KAWs (Vásconez et al. 2014). Due to a nonvanishing electric field parallel component associated with KAWs, these waves have also been considered in the problem of particle acceleration (Voitenko \& Goossens 2004; Décamp \& Malara 2006). Particle acceleration in phase-mixing of Alfvén waves in a dispersive regime has been studied both in $2 \mathrm{D}$ (Tsiklauri et al. 2005; Tsiklauri 2011) and in 3D (Tsiklauri 2012) configurations. Finally, instabilities generating KAWs in a plasma with transverse density modulations have been considered by Wu \& Chen (2013). Similar ideas involving dissipative mechanisms related to interaction of Alfvén waves or KAWs and phase-mixing have been examined in the context of the magnetospheric plasma sheet (Lysak \& Song 2011) and in coronal loops (Ofman \& Aschwanden 2002). It has been shown that ion-scale shear Alfvén waves can be excited by ion beams in the solar wind (Hellinger \& Trávníček 2011, 2013), and these can contribute to the formation of KAWs (Narivuki et al. 2014b). The possible role played by KAW-like fluctuations in heating electrons in coronal turbulence has been considered (Malara et al. 2019).

Recently, the phase-mixing of Alfvénic perturbations propagating in a pressurebalanced magnetic field transverse inhomogeneity has been numerically studied, by comparing the results of Hall-MHD and kinetic simulations (Vásconez et al. 2015; Pucci et al. 2016; Valentini et al. 2017). In these simulations the perturbation wavelength decreases, due to the interaction with the inhomogeneous background, until it reaches values of the order of the ion inertial lengh; the properties of the initial perturbation 
gradually change, eventually leading to an efficient generation of KAWs inside the inhomogeneity region. Moreover, it has been observed that such KAWs sensibly modify the initially Maxwellian ion distribution function (Vásconez et al. 2015; Valentini et al. 2017), producing temperature anisotropy, as well as localized ion beams which move along the magnetic field with a speed close to the local Alfvén velocity.

In the present paper we study a similar problem, namely, the evolution of a large-scale Alfvénic perturbation propagating in a collisionless plasma with a sheared velocity field and a uniform magnetic field parallel to the velocity. Shearing flows in plasmas with a quasi-parallel magnetic field can be found, for instance, in the interaction region between fast and slow streams of the solar wind (Bruno \& Carbone 2013), or in astrophysical jets (Hamlin \& Newman 2013). We consider an unperturbed configuration where the width of the velocity shear is of the order of few ion inertial lengths and we use a hybrid VlasovMaxwell approach to describe the system evolution. Our numerical simulations show that, also in the present case, phase-mixing acting on the Alfvénic perturbation increases the perpendicular wavenumber, until KAWs develop inside the velocity shear regions. For sufficiently large amplitudes, such waves modify the ion distribution function, locally generating a particle beam propagating along the magnetic field at the Alfvén velocity.

The plan of the paper is the following: in Section 2 we present the equations describing our model, including the form of the stationary solution and of the perturbation; in Section 3 we describe the results of the simulations; and in Section 4 we give the conclusions.

\section{The model}

We consider a collisionless, fully-ionized, magnetized plasma composed by protons and electrons. We want to describe phenomena taking place at spatial scales larger than or of the order of the proton inertial length $d_{p}=c_{A} / \Omega_{p}$ and/or of the proton Larmor radius $\rho_{p}=v_{t h, p} / \Omega_{p}$. In previous definitions, $c_{A}=B /\left(4 \pi m_{p} n\right)^{1 / 2}$ and $v_{t h, p}=\left(\kappa_{B} T_{p} / m_{p}\right)^{1 / 2}$ are respectively the Alfvén and the proton thermal speeds, related through the proton $\beta_{p}=2 v_{t h, p}^{2} / c_{A}^{2}$, while $\Omega_{p}=e B / m_{p} c$ is the proton cyclotron frequency. Moreover, $B$ is the magnetic field; $n$ is the density (assumed to be equal for protons and electrons); $T_{p}, m_{p}$ and $e$ are the proton temperature, mass and charge, respectively; $\kappa_{B}$ is the Boltzmann constant and $c$ is the speed of light.

\subsection{Equations of the model}

We employ the Hybrid Vlasov-Maxwell (HVM) model, where protons are kinetically described by the Vlasov equation, while electrons are treated as a massless fluid. As usual in the HVM approach, we assume that the electron fluid is isothermal: $T_{e}=$ const. The equations describing the HVM model, in dimensionless units, are the following:

$$
\begin{array}{r}
\frac{\partial f}{\partial t}+\mathbf{v} \cdot \nabla f+(\mathbf{E}+\mathbf{v} \times \mathbf{B}) \cdot \frac{\partial f}{\partial \mathbf{v}}=0 \\
\mathbf{E}=-\mathbf{u} \times \mathbf{B}+\frac{1}{n}(\mathbf{j} \times \mathbf{B})-\frac{1}{n} \nabla p_{e} \\
\frac{\partial \mathbf{B}}{\partial t}=-\nabla \times \mathbf{E} ; \quad \mathbf{j}=\nabla \times \mathbf{B} ; \quad p_{e}=n T_{e}
\end{array}
$$

where, $f=f(\mathbf{x}, \mathbf{v}, t)$ is the proton distribution function (DF), $n=n(\mathbf{x}, t)$ and $\mathbf{u}=\mathbf{u}(\mathbf{x}, t)$ are the density and proton bulk velocity, respectively:

$$
n(\mathbf{x}, t)=\int f(\mathbf{x}, \mathbf{v}, t) d^{3} \mathbf{v} ; \quad \mathbf{u}(\mathbf{x}, t)=\frac{1}{n(\mathbf{x}, t)} \int \mathbf{v} f(\mathbf{x}, \mathbf{v}, t) d^{3} \mathbf{v}
$$


In the above equations the magnetic field $\mathbf{B}$ is normalized to a typical value $\tilde{B}$; the density $n$ is normalized to a typical value $\tilde{n}$; velocities $\mathbf{v}$ and $\mathbf{u}$ are normalized to the typical Alfvén speed $\tilde{c}_{A}=\tilde{B}\left(4 \pi m_{p} \tilde{n}\right)^{-1 / 2}$; the electric field $\mathbf{E}$ is normalized to $\tilde{E}=\left(\tilde{c}_{A} / c\right) \tilde{B}$; the time $t$ is normalized to the typical proton gyration time $\tilde{\Omega}_{p}^{-1}$, with $\tilde{\Omega}_{p}=e \tilde{B} /\left(m_{p} c\right)$; space variables are normalized to the typical proton inertial length $\tilde{d}_{p}=\tilde{c}_{A} / \tilde{\Omega}_{p}$; the current density $\mathbf{j}$ is normalized to the value $\tilde{j}=c \tilde{B} /\left(4 \pi \tilde{d}_{p}\right)$; and the electron temperature $T_{e}$ is normalized to the typical temperature $\tilde{T}=\tilde{B}^{2} /\left(4 \pi \kappa_{B} \tilde{n}\right)$. In what follows, all the results will be expressed in terms of the above-defined dimensionless variables.

The system of equations (2.1)-(2.4) is solved by means of the HVM numerical algorithm (Valentini et al. 2007). The spatial domain is $2 \mathrm{D}$ and is defined by $D_{\mathbf{x}}=\{(x, y)\}=$ $[0, L] \times[0, L], L=16 \pi$ being the domain size, while the $3 \mathrm{D}$ domain in the velocity space is defined by $D_{\mathbf{v}}=\left\{\left(v_{x}, v_{y}, v_{z}\right),-7 v_{t h, p} \leqslant v_{i} \leqslant 7 v_{t h, p}, i=x, y, z\right\}$, where $v_{t h, p}$ is a typical dimensionless proton thermal speed, defined in the next section. Periodic boundary conditions are imposed on the boundaries of the spatial domain $D_{\mathbf{x}}$ for all quantities, while the DF is imposed to vanish at the boundaries of the velocity space domain $D_{\mathbf{v}}$. More details on the numerical method can be found in Valentini et al. (2007).

\subsection{Stationary configuration}

The stationary configuration corresponds to a magnetized plasma with a shearing flow, where the magnetic field $\mathbf{B}_{0}$ is uniform and directed parallel to the sheared bulk velocity $\mathbf{u}_{0}$. Building such kind of configuration is straightforward in the case of MHD, but it is more complex within a kinetic approach. Explicit solutions have been found by Roytershteyn \& Daughton (2008) in the fully kinetic case, and by Malara et al. (2018) within the HVM approach. Here, we will use the solution of the HVM case, which is briefly described in the following; more details can be found in Malara et al. (2018).

We consider a Cartesian reference frame, where the uniform magnetic field is directed along the $y$ axis: $\mathbf{B}_{0}=B_{0} \mathbf{e}_{y}\left(B_{0}=1\right.$ in code units), while the bulk velocity is directed along $y$ as well, but varies in the $x$ direction: $\mathbf{u}_{0}=u_{0}(x) \mathbf{e}_{y}, \mathbf{e}_{x}$ and $\mathbf{e}_{y}$ being the unit vectors in the $x$ and $y$ directions, respectively. The electric field is vanishing: $\mathbf{E}_{0}=0$, therefore protons move along helical trajectories, with the helix axis parallel to the $y$ direction. In this configuration, the motion invariants are: the particle kinetic energy $\mathscr{E}$, the parallel velocity component $v_{y}$, and the $x$-position of the particle guiding center $x_{c}=x-v_{z} / \Omega_{p},\left(\Omega_{p}=1\right.$ in code units $)$. We build a proton DF $f_{0}$ in terms of the above constants of motion, which is similar to a Maxwellian, shifted in the $v_{y}$ direction by a quantity $U\left(x_{c}\right)$, where $U(\cdot)$ is an arbitrary function which contributes to determine the profile $u_{0}(x)$ of the bulk velocity. The explicit form of the DF is given by the following combination of the motion constants:

$$
f_{0}\left(x, v_{x}, v_{y} . v_{z}\right)=\frac{n_{0}}{(2 \pi)^{3 / 2} v_{t h 0, p}^{3}} \exp \left[-\frac{1}{2 v_{t h 0, p}^{2}}\left\{v_{x}^{2}+\left[v_{y}-U\left(x-\frac{v_{z}}{\Omega_{p}}\right)\right]^{2}+v_{z}^{2}\right\}\right]
$$

where the meaning of the constants $n_{0}$ and $v_{t h 0, p}$ is specified below. Since $f_{0}$ is expressed only in terms of single-particle motion constants, it is an exact stationary solution of the Vlasov equation (2.1), provided that both $\mathbf{B}$ and $\mathbf{E}$ remain stationary. Calculating the moments of the DF, we find that the associated density is uniform: $n \equiv \int f_{0} d^{3} \mathbf{v}=n_{0}=$ const, and the bulk velocity is directed along $y: u_{0 x}=u_{0 z}=0$. As a consequence, since $T_{e}=$ const, we have $\nabla p_{e}=0$ (third equation (2.3) $)$. Moreover, being $\mathbf{u}_{0}$ parallel to $\mathbf{B}$, and $\mathbf{B}$ uniform, the generalized Ohm's law (2.2) implies that $\mathbf{E}=0$. In turn, this gives a vanishing time derivative of $\mathbf{B}$ (Faraday's law (2.3)). In summary, both the electric and 


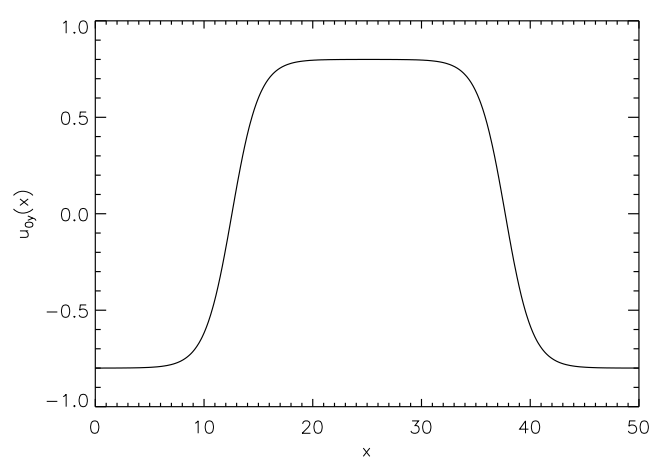

FiguRE 1. Profile of the stationary-state bulk velocity $u_{0 y}$ as a function of $\mathrm{x}$.

magnetic fields are stationary and the considered configuration represents a stationary solution for the entire set of HVM equations.

In the particular case where the function $U(\cdot)$ is constant, the expression (2.5) reduces to a shifted Maxwellian; therefore, the constant $v_{t h 0, p}$ can be interpreted as the thermal velocity of protons in regions far from the velocity shear, where the distribution function (2.5) approaches a shifted Maxwellian. The only nonvanishing bulk velocity component $u_{0 y}$ depends on the function $U(\cdot)$ through the expression (Malara et al. 2018):

$$
u_{0 y}(x)=\frac{1}{(2 \pi)^{1 / 2} v_{t h 0, p}} \int_{-\infty}^{\infty} U\left(x-\frac{v}{\Omega_{p}}\right) \exp \left(-\frac{v^{2}}{2 v_{t h 0, p}^{2}}\right) d v
$$

Therefore, the bulk velocity profile $u_{0 y}(x)$ does not coincide with $U(x)$, except in the case $U(x)=U_{0}=$ const., when $u_{0 y}(x)=U_{0}$. In general, denoting with $\Delta$ the scale of variation of the function $U(\cdot)$, it can be shown that $u_{0 y}(x) \simeq U(x)$, if $\Delta \gg R_{p}$, where $R_{p}$ is the dimensionless proton Larmor radius. Considering the opposite limit $\Delta<R_{p}$, it can also be shown that, in the considered configuration, the actual width of a velocity shear cannot be smaller than the proton Larmor radius (Malara et al. 2018).

In our case, we used the following form for the function $U(x)$ :

$$
U(x)=U_{0}\left[\tanh \left(\frac{x-L / 4}{\Delta}\right)-\tanh \left(\frac{x-3 L / 4}{\Delta}\right)-1\right]
$$

which gives a profile for $u_{0 y}(x)$ with two shears localized at $x=L / 4$ and $x=3 L / 4$. The bulk velocity is $u_{0 y} \simeq U_{0}$ in the center $x=L / 2$ of the spatial domain, while it is $u_{0 y} \simeq-U_{0}$ on the two sides $x=0$ and $x=L$. In particular, we used the following values for the above parameters: $U_{0}=0.8, \Delta=2.5$. The corresponding profile for the bulk velocity $u_{0 y}(x)$ is shown in Figure 1. The value of $U_{0}$ has been chosen relatively large to reduce the phase-mixing time, and, consequently, the computation time. On the other hand, a large jump $\Delta u_{y}$ in the bulk velocity across the shear layers can lead to the development of the Kelvin-Helmholtz $(\mathrm{KH})$ instability which would superpose to the wave dynamics. In order to prevent the $\mathrm{KH}$ instability to develop in our simulations, we have chosen $U_{0}$ sufficiently low to fulfil the condition $\Delta u_{y}<2 c_{A}$. This choice stabilizes the system against the $\mathrm{KH}$ instability, at least in the MHD case. In particular, we have chosen $\Delta u_{y} \simeq 1.6 c_{A}\left(c_{A}=1\right.$ in code units $)$.

We notice that in the two shear regions the DF (2.5) departs from a shifted Maxwellian. In particular, the proton temperature is anisotropic: $T_{\|} \geqslant T_{\perp}$, where $T_{\|}$and $T_{\perp}$ are the parallel and perpendicular temperature with respect to the magnetic field direction, 


$\begin{array}{lcccccccccc}\text { Run } & L & n_{0} & v_{t h, p} & \beta_{p} & \Delta & U_{0} & k_{0} & n_{1} & A_{1} & t_{\max } \\ 1 & 16 \pi & 1 & 1 & 2 & 2.5 & 0.8 & 2 \pi / L & 0.01 & 0.0099 & 80.0 \\ 2 & 16 \pi & 1 & 1 & 2 & 2.5 & 0.8 & 2 \pi / L & 0.1 & 0.091 & 100.0\end{array}$

TABLE 1. Values of parameters used in the runs

respectively, and agirotropy features are also present (Malara et al. 2018). Far from the velocity shears the proton temperature becomes isotropic and reaches the value $T_{0 p}$, corresponding to the asymptotic thermal speed $v_{t h 0, p}$.

\subsection{Alfvénic perturbation}

At the initial time $t=0$ a perturbation is superposed on the above stationary configuration, with properties similar to a linearly-polarized large-scale Alfvén wave. Within MHD theory, an Alfvén wave is polarized in the direction perpendicular both to the background magnetic field $\mathbf{B}_{0}$ and to the wavevector $\mathbf{k}$. In our case, $\mathbf{B}_{0}$ is in the $y$ direction, while $\mathbf{k}$ varies in time due to phase-mixing, but remaining in the $x y$ plane. Therefore, we considered a perturbation polarized along the $z$ direction. We considered a proton DF in the form: $f=f_{0}+f_{1}$, where the perturbed DF has the form of a Maxwellian shifted in the $v_{z}$ direction:

$$
f_{1}\left(y, v_{x}, v_{y}, v_{z}\right)=\frac{n_{1}}{(2 \pi)^{3 / 2} v_{t h 0, p}^{3}} \exp \left\{-\frac{v_{x}^{2}+v_{y}^{2}+\left[v_{z}-U_{z}(y)\right]^{2}}{2 v_{t h 0, p}^{2}}\right\}
$$

where $U_{z}(y)=\cos \left(k_{0} y\right)$, with $k_{0}=2 \pi / L$ corresponding to a wavelength $\lambda_{y}$ equal to the domain size $L$, and $n_{1}$ constant. We notice that $\lambda_{y} \gg d_{p}=1$. Therefore, the initial perturbation can be considered to be in an MHD regime. The total density is uniform and is given by

$$
n=\int f_{0}(x, \mathbf{v}) d^{3} \mathbf{v}+\int f_{1}(y, \mathbf{v}) d^{3} \mathbf{v}=n_{0}+n_{1}=\text { const. }
$$

This is coherent with the fact that an Alfvén wave does not involve density perturbations. The bulk velocity is given by a weighted average between the stationary state bulk velocity $\mathbf{u}_{0}$ and the bulk velocity associated with the perturbation:

$$
\mathbf{u}(x, y)=\frac{1}{n} \int \mathbf{v} f(\mathbf{x}, \mathbf{v}) d^{3} \mathbf{v}=\frac{n_{0} u_{0 y}(x) \mathbf{e}_{y}+n_{1} U_{z}(y) \mathbf{e}_{z}}{n_{0}+n_{1}}
$$

We have fixed the value $n_{0}=1$, while the parameter $n_{1}$ has been used to determine the amplitude $A_{1}=n_{1} /\left(n_{0}+n_{1}\right)$ of the initial Alfvénic perturbation.

In the MHD regime, velocity $\mathbf{u}_{1}$ and magnetic field $\mathbf{B}_{1}$ fluctuations are related by the expression $\mathbf{B}_{1}=\mp\left(B_{0} / c_{A}\right) \mathbf{u}_{1}$, where $c_{A}=1$ is the normalized Alfvén velocity associated with the equilibrium structure and the upper (lower) sign corresponds to waves propagating in the direction of $\mathbf{B}_{0}$ (opposite to $\mathbf{B}_{0}$ ). Therefore, we have chosen the magnetic field initial perturbation as:

$$
\mathbf{B}_{1}(y)=-\frac{n_{1}}{n_{0}+n_{1}} U_{z}(y) \mathbf{e}_{z}=-A_{1} U_{z}(y) \mathbf{e}_{z}
$$

Finally, the initial electric field $\mathbf{E}$ is determined by the generalized Ohm's law (2.2). 

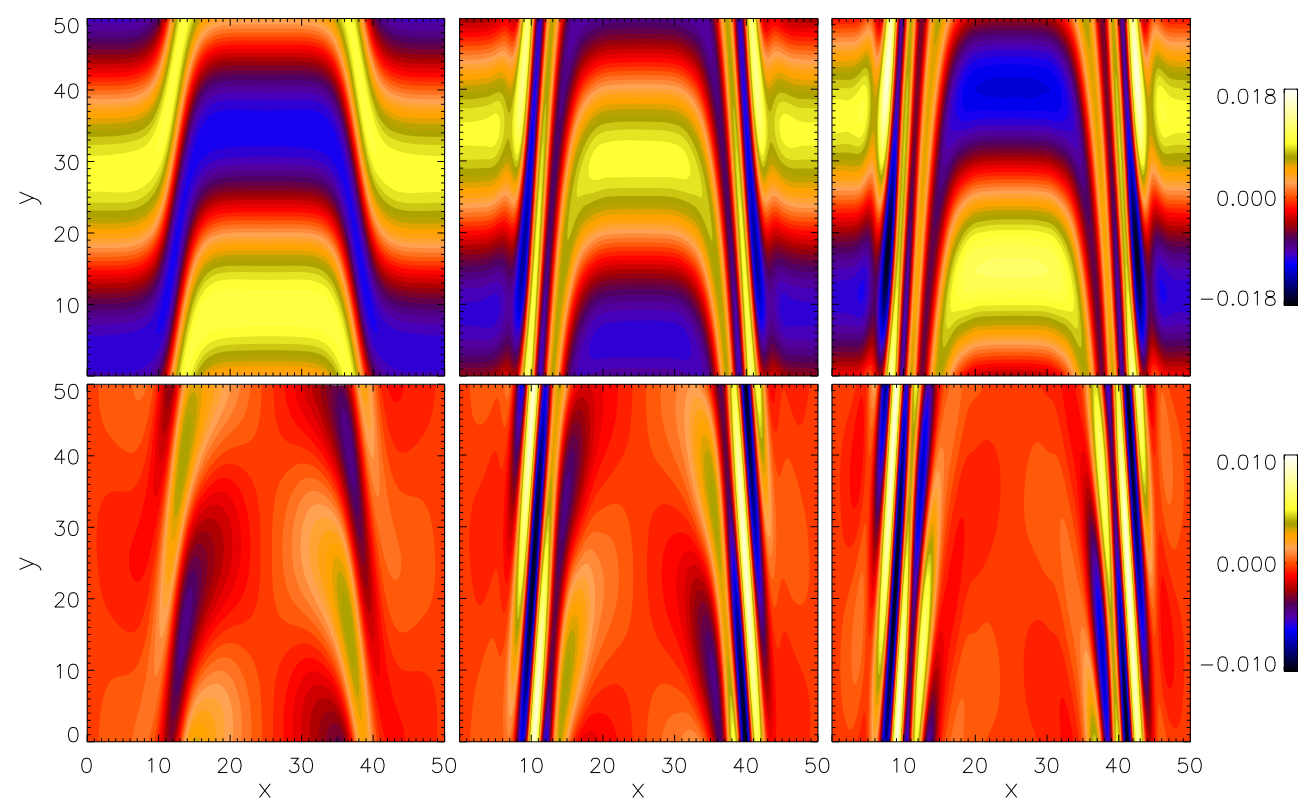

Figure 2. 2D plots in the $x y$ plane of fluctuating magnetic field components $\delta B_{z}$ (upper panels) and $\delta B_{y}$ (lower panels) at three different times: $t=20$ (left); $t=60$ (center); and $t=80$ (right). All plots refer to the low-amplitude run (Run 1).

\section{Numerical results}

We have performed numerical simulations using the HVM numerical code with the initial conditions specified in the previous section. Two simulations have been run, with two different amplitudes of the initial Alfvén wave: a low amplitude case $A_{1}=9.9 \times 10^{-3}$ (corresponding to $n_{1}=0.01$, Run 1 ) and a moderate amplitude case $A_{1}=9.1 \times 10^{-2}$ (corresponding to $n_{1}=0.1$, Run 2 ). In both runs the electron temperature is equal to the proton temperature of the stationary state far from the shear layers: $T_{e}=T_{0 p}$. The proton $\beta_{p}$ is $\beta_{p}=2 v_{t h, p}^{2} / c_{A}^{2}=2$ (i.e. $v_{t h, p}=c_{A}=1$ in code units) and the typical thermal speed is the one evaluated far from the shear regions $v_{t h, p}=v_{t h 0, p}$. We used a grid in the physical space of $N_{x} \times N_{y}$ points, with $N_{x}=512$ and $N_{y}=128$, while the grid in the velocity space has $71^{3}$ points. The maximum simulation time is indicated by $t_{\max }$ for both runs. Other parameters have been specified in the previous section and are listed in Table 1.

\subsection{Run 1: small-amplitude perturbation}

We start by describing the results obtained in the small amplitude run (Run $1, A_{1} \simeq$ 0.01). Due to the low value of the wave amplitude, nonlinear effects are negligible and the dynamics is completely dominated by the interaction of the wave with the inhomogeneity determined by the background velocity shear. Therefore, the purpose of this run is to clearly single out inhomogeneity linear effects, such as phase-mixing. In the following, the fluctuating part of any quantity $F(\mathbf{x}, t)$ is defined as $\delta F=F-\langle F\rangle_{D_{\mathbf{x}}}$, where angular parentheses indicate an average over the spatial domain $D_{\mathbf{x}}$.

In Figure 2 the fluctuating magnetic field component $\delta B_{z}$ is plotted for three different times during the simulation (upper panels). At the initial time (not shown), only the $\delta B_{z}$ component, corresponding to the initial Alfvénic perturbation, is nonvanishing. 

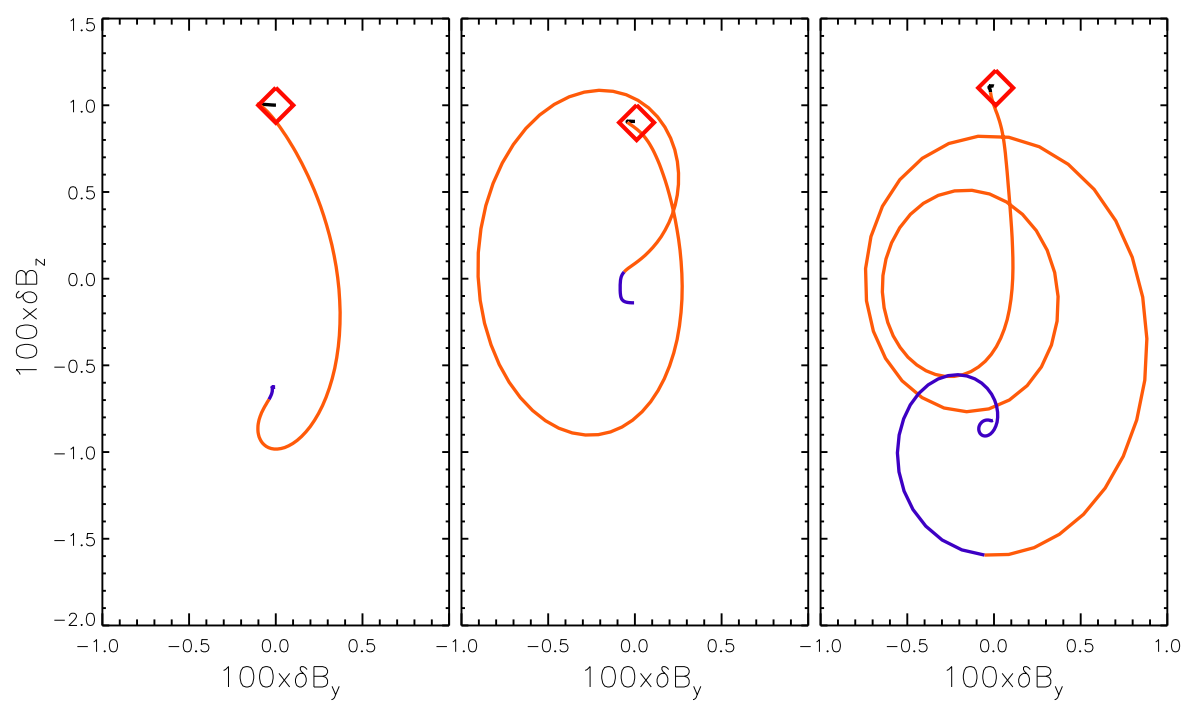

FiguRe 3 . Hodograms in the $\delta B_{y}-\delta B_{z}$ plane, relative to Run 1 , at time $t=t_{1}=20$ along the line $L / 2 \leqslant x \leqslant L, y=y_{1}=10$ (left panel); at time $t=t_{2}=40$ along the line $L / 2 \leqslant x \leqslant L$, $y=y_{2}=45$ (center panel); and at time $t_{3}=80$ along the line $L / 2 \leqslant x \leqslant L, y=y_{3}=15$ (right panel). Black, orange and blue sections are relative to the intervals $L / 2 \leqslant x<31,31 \leqslant x<44.5$, and $44.5 \leqslant x \leqslant L$, respectively. The red diamond indicate the point at $x=L / 2$.

The effects of phase-mixing on the time evolution of $\delta B_{z}$, due to space variations of the bulk velocity $u_{y}$, are clearly visible. The wave propagation velocity $\mathbf{v}_{W}$ in the simulation reference frame is the sum of the Alfvén velocity plus the bulk velocity $\mathbf{v}_{W}(x)=\left[c_{A}+u_{y}(x)\right] \mathbf{e}_{y}$. Accordingly, $v_{W}$ is larger in the center of the spatial domain than on the two sides. As a consequence, initially plane wavefronts are progressively bent in the two velocity shear regions, where the wavevector perpendicular component $k_{x}$ locally increases, while the wavelength decreases. Dispersive effects become effective for wavevectors of the order of $k_{\text {disp }} \simeq d_{p}^{-1}=1$, corresponding to a wavelength $\lambda_{\text {disp }} \simeq 2 \pi$. At later times in the simulation $(t \gtrsim 60)$, the wavelength of the perturbation in the shear regions has decreased to $\lambda \sim \lambda_{\text {disp }}$. After that time, dispersive effects becomes relevant, at least within the velocity shear regions. In these regions, we observe that the magnetic fluctuation parallel component $\delta B_{y}$ (shown in Figure 2, lower panels) increases in time, until reaching values of the same order of $\delta B_{z}$ for times $t \geqslant 60$. In the MHD case, where dispersive effects are neglected, the same phase-mixing problem would give a null $\delta B_{y}$ for all times, implying that the generation of $\delta B_{y}$ is only caused by non-MHD dispersive effects. In fact, $\delta B_{y}$ grows only in the two shear regions, where the wavelength is small enough to make dispersive effects relevant. In summary, in the velocity shear regions perturbations change their properties: the initial linear Alfvénic polarization is modified by the growth of a parallel component $\delta B_{y}$, the wavevector becomes nearly perpendicular $\left(k_{\|} \ll k_{\perp}\right)$ and of the order of the inverse proton inertial length. These evidences suggest that the initial Alfvénic perturbation has been locally converted into a KAW.

In order to have a clear identification of the perturbations generated in the velocity shear regions, we have first considered the group velocity $\mathbf{v}_{g}$ of the waves. Vásconez et al. (2015) have shown that in the dispersive MHD all wave modes have a nonvanishing com- 
ponent of $v_{g \perp}$ in the direction perpendicular to $\mathbf{B}_{0}$. In particular, for fast magnetosonic waves it is $v_{a \perp} \geq c_{A}$, while for slow magnetosonic and Alfvén waves it is $v_{g \perp} \ll c_{A}$ (Vásconez et al. 2015). In our simulation, we noticed that fluctuations generated in the shear regions propagate also perpendicularly to $\mathbf{B}_{0}$, in the direction from the center to the lateral parts of the spatial domain. However, we verified that the transverse propagation speed is one order of magnitude lower than the Alfvén speed. We conclude that such fluctuations cannot belong to the fast magnetosonic branch.

To further discriminate among slow and Alfvénic fluctuations, we have examined how the orientation of $\delta \mathbf{B}$ varies along segments parallel to the $x$ axis. In the velocity shear regions these segments are quasi-parallel to the wavevector direction. An example is given in Figure 3, where three hodograms are plotted: $\delta B_{z}$ vs $\delta B_{y}$. These are calculated at three different times: the left panel refers to the segment defined by $L / 2 \leqslant x \leqslant L$, $y=y_{1}=10$ at time $t_{1}=20$, the center panel to the segment $L / 2 \leqslant x \leqslant L, y=y_{2}=45$ at time $t_{1}=40$, while the right panel to the segment $L / 2 \leqslant x \leqslant L, y=y_{3}=15$ at time $t_{3}=80$. The values $y_{1}, y_{2}$ and $y_{3}$ have been chosen such that $\delta B_{z}\left(x=L / 2, y_{1,2,3}, t_{1,2,3}\right) \simeq$ $\max \left\{\delta B_{z}\left(x=L / 2, y, t_{1,2,3}\right), 0 \leqslant y \leqslant L\right\}$. Different sections of the segments are indicated by different colors: black corresponds to the central homogeneous region $(L / 2 \leqslant x<31)$; orange corresponds to the shear region $(31 \leqslant x<44.5)$; blue corresponds to the lateral homogeneous region $(44.5 \leqslant x \leqslant L)$. The diamond indicates the values of $\delta B_{y}$ and $\delta B_{z}$ at the position $x=L / 2$.

Figure 3 can be used to determine the polarization of the magnetic perturbation in the $\delta B_{y^{-}} \delta B_{z}$ plane, which is nearly perpendicular to the wavevector in the shear region. The left panel of Figure 3 refers to an early stage of time evolution. At that time, in the shear region (orange section of the curve) the wave polarization is still essentially linear, $z$-aligned, as the initial Alfvén wave. In fact, since the perturbation wavelength in the shear region is still larger than $d_{p}$ (see Figure 2, left panels), dispersive effects are not large enough to sensibly modify the initial polarization. In the same hodogram we also notice very small variations of the perturbation in the two homogeneous regions (black and blue sections of the curve); this is due to the fact that the hodogram is drawn along a segment that, in the homogeneous regions, is quasi parallel to wavefronts.

This latter feature is present also at the time $t=40$ (central panel of Figure 3), but now the polarization in the shear region (orange section) has turned into clockwise elliptical. This polarization change is due to dispersive effects: they are now more relevant in consequence of the wavelength decrease induced by phase-mixing. The same polarization is also found in the shear region at $t=80$ (right panel); the orange section of the curve has a larges number of turns with respect to $t=40$ because phase-mixing has further decreased the perturbation wavelength. Hodograms calculated along other parallel segments, which are not shown here, display the same behaviour. Considering wave modes within the dispersive MHD (Vásconez et al. 2015), Alfvén and fast magnetosonic waves are both clockwise-elliptically polarized, while slow magnetosonic waves are counterclockwise polarized. We can therefore exclude that fluctuations generated in the shear regions during system evolution belong to the slow magnetosonic branch.

On the base of the above considerations about polarization and group velocity, the perturbations generated in the velocity shear regions belong to the Alfvén branch, i.e., they are KAWs. Therefore, the phase-mixing mechanism acting in the shear regions gradually triggers a mode conversion from the initial Alfvén wave to KAW fluctuations. Finally, we notice that a small amplitude clockwise-elliptically polarized fluctuation is also present in the lateral homogeneous region at time $t=80$ (Figure 3, right panel, blue section); due to the nonvanishing $v_{g \perp}$, KAWs do not remain confined into the shear regions but tend to move to the side homogeneous regions. 


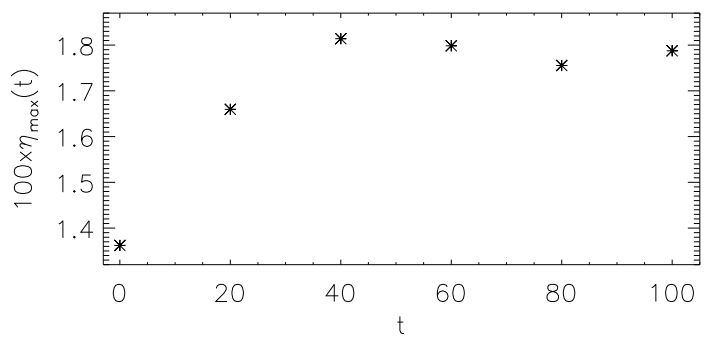

FIgURE 4 . The quantity $\eta_{\max }$ is plotted as a function of time $t$, for the moderate-amplitude run (Run 2).

\subsection{Run 2: moderate-amplitude perturbation}

When increasing the initial perturbation amplitude, the effects of fluctuating fields on the proton DF become more relevant. In order to study such kinetic effects, being at the same time able to identify the characteristics of the propagation of KAWs during the system evolution, in Run 2 we have chosen a moderately larger amplitude of the initial perturbation $n_{1}=0.1$, corresponding to $A_{1}=0.091$. A larger amplitude would produce significant deformations of the initial equilibrium configuration, making hard to clearly identify the nature of the transverse fluctuations produced by phase-mixing.

The time evolution of magnetic and velocity fields in Run 2 is qualitatively similar to that observed in Run 1 (see Figure 2). In particular, the initial Alfvén wave undergoes phase-mixing in the two velocity shear regions, locally generating small-scale perturbations with a quasi-perpendicular wavevector. Such perturbations are elliptically polarized in the sense of the Alfvén dispersive branch and slowly expand in the transverse direction, moving outside of the shear regions. Therefore, also in this moderate-amplitude case we can conclude that the initial Alfvén wave is converted into a KAW-like fluctuation by the interaction with the background inhomogeneity due to the velocity shear.

The main differences between the small and moderate-amplitude cases consist in the modifications of the proton distribution function induced by fluctuations, which are more relevant for Run 2. In order to give a quantitative measure for such a phenomenon, we define the quantity $\eta$ :

$$
\eta(\mathbf{x}, t)=\frac{1}{n(\mathbf{x}, t)} \sqrt{\int_{D_{\mathbf{v}}}\left[f(\mathbf{x}, \mathbf{v}, t)-f_{0}(\mathbf{x}, \mathbf{v})\right]^{2} d^{3} \mathbf{v}}
$$

which represents the $L^{2}$ norm of the departure (in velocity space) between the proton DF $f$, at a position $\mathbf{x}$ and time $t$, and the unperturbed DF $f_{0}$ at the same position, normalized to the density. Since $\eta$ is a positive-definite quantity, it is useful to consider also its maximum value calculated over the spatial domain, at a given time $t: \eta_{\max }(t)=\max _{D_{\mathbf{x}}}\{\eta(\mathbf{x}, t)\}$. The corresponding position is indicated by $\mathbf{x}_{M}(t)$ : $\eta\left(\mathbf{x}_{M}(t), t\right)=\eta_{\max }(t)$.

In Figure 4 the quantity $\eta_{\max }(t)$ is plotted as a function of time, for Run 2 . At the initial time it is $\eta \neq 0$, due to the DF initial perturbation $f_{1}$ which is superposed on the stationary DF $f_{0}$. We can see that the departure of the DF $f$ from $f_{0}$ slightly increases in time, indicating a progressive modification of the DF due to the effects of the perturbation. The growth of $\eta_{\max }(t)$ saturates at the time $t \simeq 40$, remaining roughly constant after that time. The saturation value is $\eta_{\max \text { sat }} \simeq 1.8 \times 10^{-2}$, which is $\simeq 1.3 \eta_{\max }(t=0)$. The relatively low increase of $\eta_{\max }$ is partially due to the moderate 


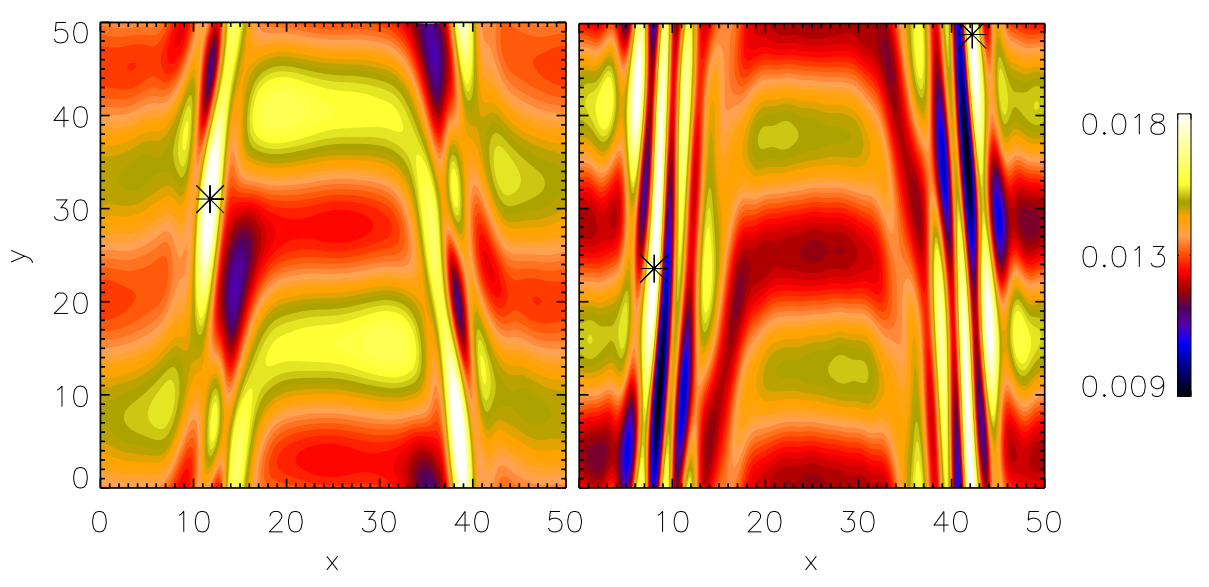

Figure 5. 2D plots of the quantity $\eta(\mathbf{x}, t)$ in the $x y$ plane, at time $t=40$ (left panel) and $t=100$ (right panel), for the moderate-amplitude run (Run 2). Asterisks indicate the positions where $\eta$ is maximum at the given time.

amplitude of the perturbation and partially to the large value of $\beta_{p}$. In fact, larger values of $\beta_{p}$ corresponds to larger thermal energy in the proton population, when compared to the energy associated with the perturbation. We expect to find an increase of $\eta_{\max }$ more relevant than in the present case, when larger perturbation amplitudes and/or lower $\beta_{p}$ are considered.

In Figure 5, 2D plots of $\eta(\mathbf{x}, t)$ in the $x y$ plane are shown at times $t=40$ and $t=100$. Asterisks indicate the location $\mathbf{x}_{M}$ where $\eta$ attains the maximum value $\eta_{\max }$ for the given time. From this figure we see that the largest departures from the background DF $f_{0}$ are mainly localized within the velocity shear regions, where the smallest wavelengths form in the perturbation. The spatial structure of $\eta(\mathbf{x}, t)$ appears to be influenced by phase-mixing, indicating that the DF is also spatially modulated on a small transverse scale. Therefore, departures from the unperturbed proton DF are strictly related to the generation of small scales, of the order of $d_{p}$.

$3 \mathrm{D}$ iso-surface plots of the proton $\mathrm{DF} f\left(\mathbf{x}_{M}(t), \mathbf{v}, t\right)$ in velocity space are shown in Figure 6 for $t=40$ and $t=100$, at the position $\mathbf{x}_{M}(t)$. For comparison, the unperturbed DF $f_{0}\left(\mathbf{x}_{M}(t), \mathbf{v}\right)$ is plotted at the same position (Figure 6 left panels). The DF modifications become evident by comparing $f$ and $f_{0}$. At time $t=40$, such variations appear as a modulation in form of rings on the considered isosurface (Figure 6, upperright panel), which are co-axial with the direction $y$ of the background magnetic field $\mathbf{B}_{0}$, indicated by a blue arrow. We can also notice that the unperturbed DF $f_{0}$ departs from a Maxwellian, the parallel temperature $T_{p \| \mid}$ being larger than the perpendicular one $T_{p \perp}$ (Malara et al. 2018). At time $t=100$, the DF $f$ shows a peculiar feature where $\eta=\eta_{\text {max }}$; namely, a "bulge" is present in the isosurface (Figure 6, lower-right panel), indicating the presence of a sub-population of protons moving along $\mathbf{B}_{0}$ faster than the core particles. The existence of this beam of accelerated protons can also be seen in Figure 7 , where a 

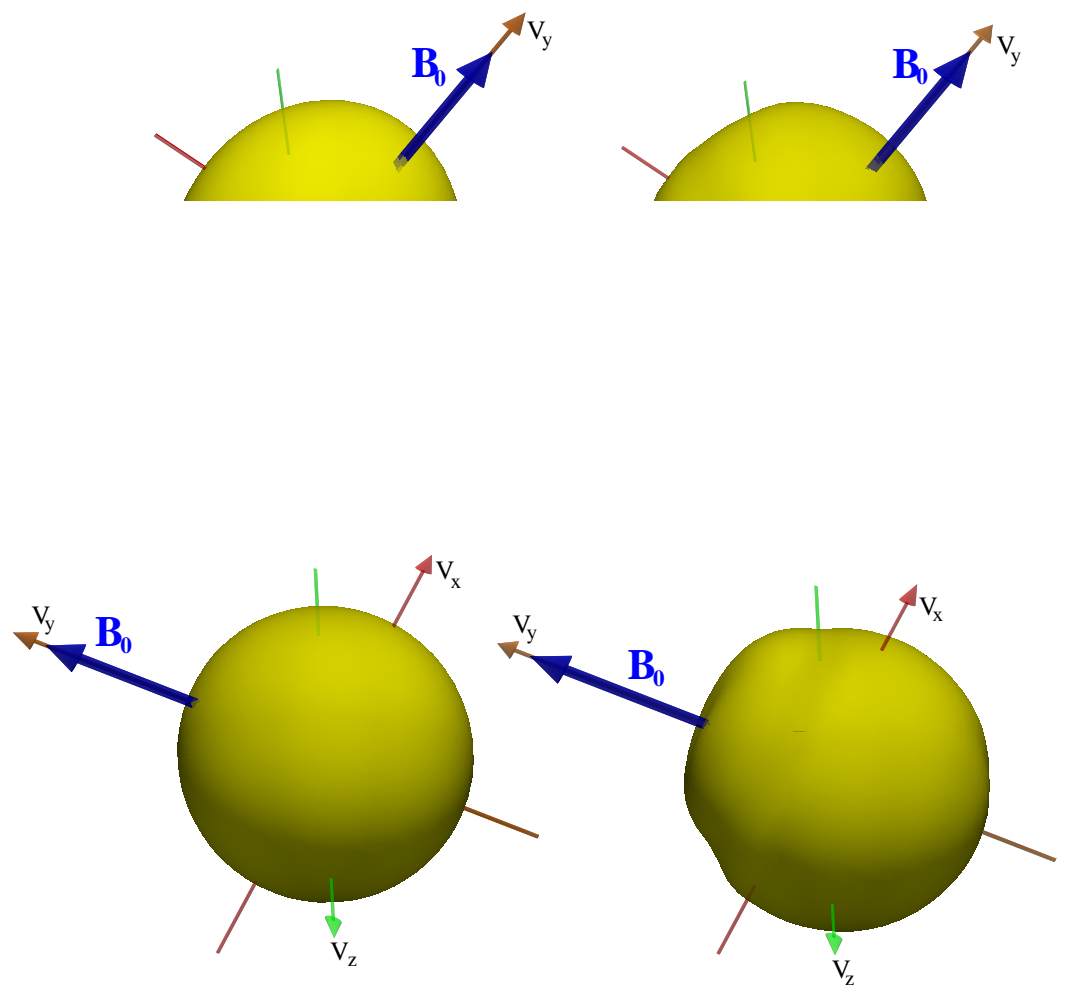

Figure 6. Isosurfaces of the 3D proton velocity DF $f\left(\mathbf{x}_{M}(t), \mathbf{v}, t\right)$ (right panels), and of the stationary DF $f_{0}\left(\mathbf{x}_{M}(t), \mathbf{v}\right)$ (left panels), at time $t=40$ (upper panels) and $t=100$ (lower panels), calculated in the spatial position $\mathbf{x}_{M}(t)$ where $\eta=\eta_{\max }$ at the given time. Isosurfaces are relative to the values $f=f_{0}=0.008$. Plots refer to the moderate-amplitude run (Run 2).

"cut" of the DF shown in Figure 6 for $t=100$ is plotted in velocity space as a function of $v_{y}$, for $v_{x}=v_{z}=0$ (black line). In the same figure, the corresponding profile of the unperturbed DF $f_{0}$ is plotted for comparison (orange line), along with the difference $f-f_{0}$ of the two profiles (blue line). The proton beam is evident also in Figure 7 in particular, the difference in velocity between the beam and core population along the $\mathbf{B}_{0}$ direction is $\Delta v_{y} \simeq c_{A}=1$.

Concerning the origin of the beam, we observe that KAWs are characterized by a component of the electric field $E_{y}$ parallel to the background magnetic field $\mathbf{B}_{0}$ (e.g., Hollweg 1999). Such a component is vanishing in the limit of ideal MHD and can be responsible for particle energization. In Figure 8 a 2D plot of the parallel electric field component $E_{y}$ at the time $t=100$ is shown. Figure 8 shows that $E_{y}$ is localized in the region where the smallest spatial scales are present in the perturbation, while it is negligible outside. Moreover, the spatial pattern of $E_{y}$ is similar to that of other perturbed quantities, and the wavelength in the parallel $y$ direction is $\lambda_{\|}=2 \pi / k_{0}=L$. As a consequence, we can define a sort of electric potential $\phi$, such as $E_{y}=-\partial \phi / \partial y$. Of course, the dependence of $\phi$ on $y$ is also periodic, with a period equal to $L$. Protons, in their motion, feel this periodic potential and can interact with it. In particular, part of the ion population can remain trapped into the potential well associated with the spatially-modulated $E_{y}$ component. For a given particle kinetic energy, this trapping is more probable for protons which move quasi-parallel to $\mathbf{B}_{0}$, i.e., with $v_{x}, v_{z} \ll v_{y}$; this latter condition corresponds to the cut in Figure 7 . The potential well co-moves with the 


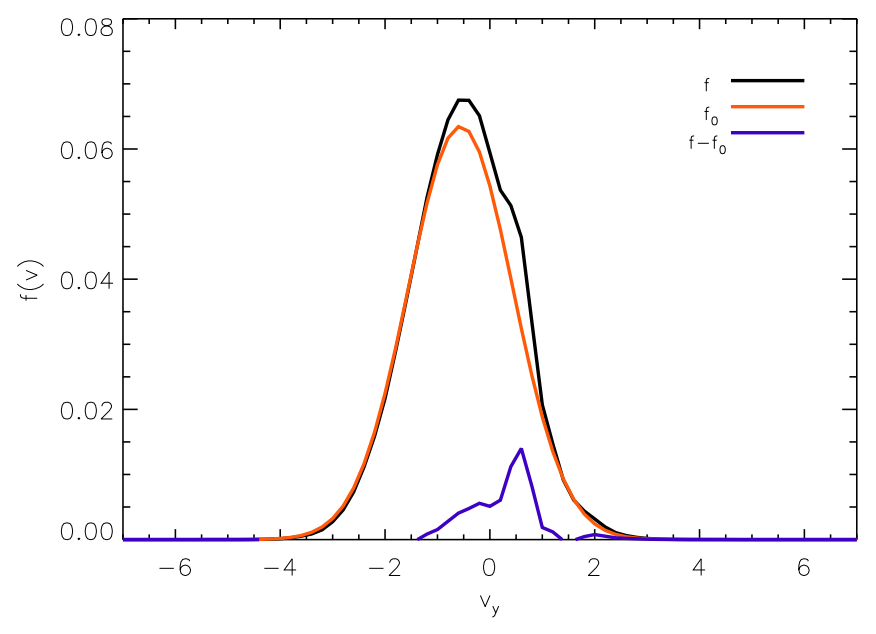

Figure 7. Profiles of the proton distribution functions $f$ (black line), $f_{0}$ (orange line), and of the difference $f-f_{0}$ (blue line) are plotted as functions of $v_{y}$, for $v_{x}=v_{z}=0$, at the space position $\mathbf{x}_{M}(t)$ at time $t=100$. Plots refer to the moderate-amplitude run (Run 2).

wave along the magnetic field with a velocity $\simeq c_{A}$ in the plasma bulk reference frame, which explains why trapped protons have an average velocity of $u_{y}(x)+c_{A}$. This process can account for the formation of the DF features observed in Figure 7.

Indeed, in previous simulations of Alfvén wave phase-mixing generated by magnetic field inhomogeneities, the presence of ion beams moving along the magnetic field at the local Alfvén speed has been observed by Vásconez et al. (2015); Valentini et al. (2017). These authors have shown that the origin of such beams is the parallel electric field associated with KAW-like fluctuations, generated in the inhomogeneity regions by the phase-mixing mechanism. It is likely that the same physical process is responsible for the creation of suprathermal ion beams also in the case considered in the present paper.

\section{Conclusions}

In this paper we have studied the evolution of an Alfvén wave in a collisionless plasma, propagating in a stationary configuration characterized by a sheared flow with a uniform magnetic field parallel to the velocity field. The study has been carried out numerically, by means of the HVM code. We considered a width of the shear layers of the order of the proton inertial length $d_{p}$. In order to properly describe the unperturbed configuration, we used an exact analytical solution suitable for the HVM approach (Malara et al. 2018). The physical mechanism acting on the time evolution of the perturbation is phase-mixing, induced by transverse variations of the plasma bulk velocity, which bends wavefronts and generates increasingly small scales in the transverse direction. Though the initial perturbation is in a MHD regime, i.e., the wavelength $\lambda$ is initially much larger than proton scales, later in time $\lambda$ locally becomes of the order of $d_{p}$. Once this stage is reached, both dispersive and kinetic effects come into play and the initial Alfvén wave is converted into a KAW. The identification of the perturbation within the shear region as a KAW has been done considering both its polarization, which is turned from linear into clockwise-elliptical, and the transverse group velocity, which is nonvanishing but much less than the Alfvén speed.

In the moderate amplitude run, properly kinetic effects have been observed, such 


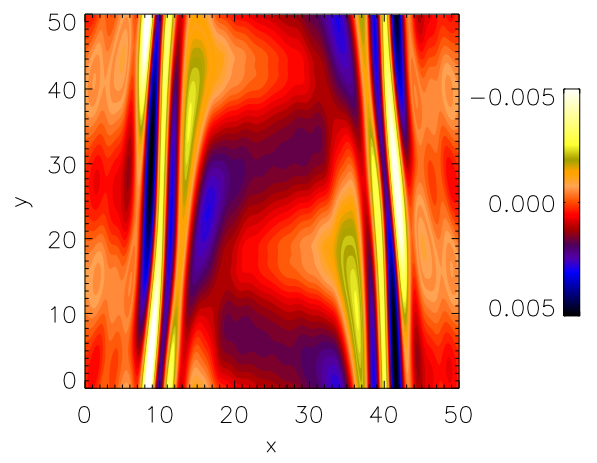

FiguRE 8. 2D plot of the electric field parallel component $E_{y}$ in the $x y$ plane, calculated at time $t=100$ for the moderate-amplitude run (Run 2).

as modifications of the initial stationary distribution function. In the considered case, variations of the DF are not particularly large, due both to the moderate perturbation amplitude and to the large value of the proton beta parameter $\left(\beta_{p}=2\right)$; in fact, larger values of $\beta_{p}$ correspond to a lower ratio of the perturbation energy to the bulk thermal energy. We decided not to increase further the amplitude of the initial perturbation as for larger amplitudes the wave features of the KAW are no longer easily identified.

Nevertheless, an interesting feature has been observed, namely, the generation of a beam of suprathermal protons moving along the magnetic field with a speed given by the sum of the Alfvén velocity and the local bulk velocity. The existence of such a beam can be related to an electric field component $\delta E_{\|}$parallel to the magnetic field, which characterizes KAWs, and which has been indeed observed in our simulations in the velocity shears regions, where KAWs are generated. We notice that the presence of magnetic-field aligned proton beams, is often observed in the solar-wind plasma (Marsch 2006). Therefore, our results suggest that such a feature could be related to the presence of KAW-like fluctuations in the solar wind.

Most of the behaviour and features observed in the present simulations have been also found in another situation in which an Alfvén wave propagate in a background configuration where the magnetic field $\mathbf{B}_{0}$ is inhomogeneous, with spatial variations perpendicular to $\mathbf{B}_{0}$, at scales comparable with the proton inertial length (Vásconez et al. 2015; Pucci et al. 2016; Valentini et al. 2017). In that case, phase-mixing acting on the Alfvén wave is due to spatial variations of the Alfvén velocity. The results found in the latter studies and these obtained in the present investigation can be considered to be complementary. We can conclude that the observed phenomenology is due to phasemixing at proton scales, regardless of the origin of such a process (magnetic field and/or bulk velocity inhomogeneities); in other words, as long as the propagation velocity of Alfvén waves is spatially inhomogeneous in the direction perpendicular to the background field, these can be successfully converted into KAWs.

As a final remark, we observe that the present results have been derived within a simplified configuration, where it is possible to distinguish a single well-defined wave from the inhomogeneous background. Nevertheless, our results give some indications on the possibility of generating KAWs in more complex contexts, like in a turbulent setting. In fact, the wave-inhomogeneity coupling considered in this investigation is of a similar nature as the nonlinear coupling between fluctuations which generate the turbulent cascade in MHD: in a turbulent regime fluctuations with two different $k$ s couple to 
produce a third one at higher $k$, while in the configuration here considered a fluctuation with a well defined $k$ couples with the $k$ coming from the inhomogeneity.

We therefore conclude that the phenomenon we studied is closely related to the mechanism that favors perpendicular spectral transfer in the nonlinear cascade (e.g., Shebalin et al. 1983). Moreover, our results give a positive indication about the possibility of generating KAW-like fluctuations at proton scales within a turbulent cascade, as suggested by solar wind observations (Bale et al. 2005; Sahraoui et al. 2012).

\section{Acknowledgements}

The authors are grateful to Prof. W.H. Matthaeus for many useful discussions about the subject of the paper. The work by F. Pucci has been supported by Fonds Wetenschappelijk Onderzoek Vlaanderen (FWO) through the postdoctoral fellowship 12X0319N. This paper has received funding from the European Unions Horizon 2020 research and innovation program under Grant Agreement No. 776262 (AIDA, www.aida-space.eu)

\section{REFERENCES}

Araneda, J. A., Marsch, E. \& Viñas, A. F. 2008 Proton core heating and beam formation via parametrically unstable Alfvén-cyclotron waves. Phys. Rev. Lett. 100 (12), 125003.

Belcher, J. \& Davis, L. 1971 Large-amplitude Alfvén waves in the interplanetary medium. J. Geophys. Res. 76 (16), 3534-3563.

Bale, S., Kellogg, P., Mozer, F., Horbury, T. \& Reme, H. 2005 Measurement of the electric fluctuation spectrum of magnetohydrodynamic turbulence. Phys. Rev. Lett. 94 (21), 215002

Bale, S., Badman, S.T, Bonnell, J.W., Bowen, T.A., Burgess, D., Case, A.W. et al. 2019 Highly structured slow solar wind emerging from an equatorial coronal hole. Nature 576 (21), 237-242

Bruno, R. \& Carbone, V. 2013 The solar wind as a turbulence laboratory. Living Rev. Sol. Phys 10, 2.

Califano, F., Chiuderi, C. \& Einaudi, G. 1990 Nonresonant resistive dissipation of incompressible magnetohydrodynamic waves. Astrophys. J. 365, 757-763.

Califano, F., Chiuderi, C. \& Einaudi, G. 1992 Nonresonant resistive dissipation of compressible magnetohydrodynamic waves. Astrophys. J. 390, 560-566.

Carbone, V. \& Veltri, P. 1990 A shell model for anisotropic magnetohydrodynamic turbulence. Geophys. Astrophys. Fluid Dyn. 52 (1-3), 153-181.

Carbone, V., Malara, F. \& Veltri, P. 1995 A model for the three-dimensional magnetic field correlation spectra of low-frequency solar wind fluctuations during Alfvénic periods. J. Gephys. Res. 100 (A2) 1763-1778.

Cerri, S.S., Kunz, M.W, \& Califano, F. 2018 Dual phase-space cascades in 3D hybrid Vlasov-Maxwell turbulence. Astrophys. J. Lett.,856, L13.

Chen, C., Boldyrev, S., Xia, Q. \& Perez, J. 2013 Nature of subproton scale turbulence in the solar wind. Phys, Rev. Lett. 110 (22), 225002.

Dasso, S., Milano, L. J., Matthaeus, W. H. \& Smith, C. W. 2005 Anisotropy in Fast and Slow Solar Wind Fluctuations. Astrophys. J. 635 (2) L181-L184.

DAvilA, J. M. 1987 Heating of the solar corona by the resonant absorption of Alfvén waves. Astrophys. J. 317, 514-521.

DÉCAmP, N. \& Malara, F. 2006 Electron acceleration in turbulent coronal loops by kinetic Alfvén wave, in SOHO-17. 10 Years of SOHO and Beyond vol. 617, p. 26., ESA Publ. Div., Noordwijk, Netherlands.

Gary, S. P., \& Nishimura, K. 2004 Kinetic Alfvén waves: Linear theory and a particle-in-cell simulation. J. Geophys. Res. 109, A02109.

Ghosh, S., Matthaeus, W. H., Roberts, D. A. \& Goldstein, M. L. 1998 The evolution of slab fluctuations in the presence of pressure-balanced magnetic structures and velocity shears. J. Geophys. Res. 103 (A10), 23,691-23,704. 
Goldstein, B. E., Neugebauer, M., Zhang, L. D. \& Gary, S. P. 2000 Observed constraint on proton-proton relative velocities in the solar wind. Geophys. Res. Lett. 27 (1), 53-56

Goodrich, C. \& Lazarus, A. 1976 Suprathermal protons in the interplanetary solar wind. J. Geophys. Res. 81 (16), 2750-2754.

Hamlin, N. D. \& Newman, W. I. 2013 Role of the Kelvin-Helmholtz instability in the evolution of magnetized relativistic sheared plasma flows. Phys. Rew. E 87, 043101.

Hellinger, P., Trávníček, P., Kasper, J. C., \& Lazarus, A. J. 2006 Solar wind proton temperature anisotropy: Linear theory and WIND/SWE observations. Geophys. Res. Lett. 33, L09101.

Hellinger, P. \& TrÁvníčEK, P. 2011 Proton core-beam system in the expanding solar wind: hybrid simulations. J. Geophys. Res. 116, A11101.

Hellinger, P. \& TrávníčEK, P. 2013 Protons and alpha particles in the expanding solar wind: hybrid simulations. J. Geophys. Res. Space Phys. 117, 54215430.

HollweG, J. V. 1987 Resonance absorption of magnetohydrodynamic surface waves physical discussion. Astrophys. J. 312, 880-885.

Hollweg, J. V. 1999 Kinetic Alfvén wave revisited. J. Geophys. Res. 104 (A7), 14,811-14,819.

Howes, G. G., Dorland, G. W., Cowley, S., Hammett, G. W., Quataert, E., Schekochinin, A. A. \& Tatsuno, T. 2008a Kinetic simulations of magnetized turbulence in astrophysical plasmas. Phys. Rev. Lett. 100 (6), 65004.

Howes, G. G., S. C. Cowley, W. Dorland, G. W. Hammett, E. Quataert, and A. A. ScheKochinin 2008b A model of turbulence in magnetized plasmas: implications for the dissipation range in the solar wind. J. Geophys. Res. 113, A05103.

Kappraff, J. \& Tataronis, J. 1977 Resistive effects on Alfvén wave heating. J. Plasma Phys. 18 (02), 209-226.

KaghashviLI, E.K. 1999 On the acceleration of the solar wind: Role of the inhomogeneous flow. Astrophys. J. $\mathbf{5 1 2}$ (2), 969-974.

Kiyani, K. H., Chapman, S. C., Sahraoui, F., Hnat, B., Fauvarque, O. \& Khotyaintsev, Y. V. 2013 Enhanced magnetic compressibility and isotropic scale invariance at sub-ion larmor scales in solar wind turbulence. Astrophys. J. 763 (1), 10.

Landi, S., Velli, M. \& Einaudi, G. 2005 Alfvén waves and shock wave formation at an x-point magnetic field configuration. Astrophys. J. 624 (1), 392-401.

Lee, M. \& Roberts, B. 1986 On the behavior of hydromagnetic surface waves. Astrophys $J$. 301, 430-439.

LySAK, R. L. \& Song, Y. 2011 Development of parallel electric fields at the plasma sheet boundary layer. J. Geohys. Res. 116, A00K14.

Malara, F., Veltri, P., Chiuderi, C. \& Einaudi, G. 1992 Incompressible disturbances in nonuniform media-formation of small scales. Astrphys. J. 396, 297-310.

Malara, F., Primavera, L. \& Veltri, P. 1996a Compressive fluctuations generated by time evolution of Alfvénic perturbations in the solar wind current sheet. J. Geophys. Res. 101 (A10), 21,597-21,617.

Malara, F., Primavera, L. \& Veltri, P. 1996b Formation of small scales via Alfvén wave propagation in compressible nonuniform media. Astrophys. J. 459, 347-364.

Malara, F., Petkaki, P. \& Veltri, P. 2000 Dissipation of Alfvén waves in force-free magnetic fields: Competition between phase mixing and three-dimensional effects. Astrophys. $J$. 533, $523-534$.

Malara, F., De Franceschis, M. F. \& Veltri, P. 2003 Alfvén wave propagation and dissipation in a 3D-structured compressible plasma. Astron. Astrophys. 412 (2), 529-539.

Malara, F., De Franceschis, M. F. \& Veltri, P. 2005 Dissipation of Alfvén waves in complex 3D coronal force-free structures. Astron. Astrophys. 443 (3), 1033-1046.

Malara, F., De Franceschis, M. F. \& Veltri, P. 2007 Alfvén wave dissipation and topological properties of 3D coronal force-free magnetic fields. Astron. Astrophys. 467 (3), 1275-1284.

Malara, F., Pezzi, O. \& Valentini, F. 2018 Exact hybrid Vlasov equilibria for sheared plasmas with in-plane and out-of-plane magnetic field. Phys. Rev. E 97, 053212.

Malara, F., Nigro, G., Valentini, F. \& Sorriso-Valvo, L. 2019 Electron heating by kinetic Alfvén waves in coronal loop turbulence. Astrophys. J. 871, 66.

Marsch, E., Mühlhäuser, K.-H., Schwenn, R., Rosenbauer, H., Pilipp, W. \& 
Neubauer, F. 1982 Solar wind protons: three-dimensional velocity distributions and derived plasma parameters measured between 0.3 and 1 AU. J. Geophys. Res. 87 (A1), $52-72$

Marsch, E. 2006 Kinetic Physics of the Solar Corona and Solar Wind. Living Rev. Solar Phys. 3,1 .

Matteini, L., Landi, S., Velli, M. \& Hellinger, P. 2010 Kinetics of parametric instabilities of Alfvén waves: Evolution of ion distribution functions. J. Geophys. Res. 115, A09106

Matthaeus, W. H., Goldstein, M. L. \& King, J. 1986 An interplanetary magnetic field ensemble at 1 AU. J. Geophys. Res. 91 (A1), 59-69.

Matthaeus, W. H., Goldstein, M. L. \& D. A. Roberts, J. 1990 Evidence for the presence of quasi-two-dimensional nearly incompressible fluctuations in the solar wind. J. Geophys. Res. 95 (A12), 20,673-20,683.

Matthaeus, W. H., Servidio, S., Dmitruk, P., Carbone, V., Oughton, S., Wan, M. \& Osman, K. T. 2012 Local Anisotropy, Higher Order Statistics, and Turbulence Spectra. Astrophys. J. $\mathbf{7 5 0}$ (2), 103.

McLaughlin, J., Hood, A., \& De Moortel, I. 2011 Review article: MHD wave propagation near coronal null points of magnetic fields. Space Sci. Rev. 158 (2-4), 205-236.

Milano, L. J. Matthaeus, W. H., Dmitruk, P. \& Montgomery, D. C. 2001 Local anisotropy in incompressible magnetohydrodynamic turbulence. Phys. Plasmas 8 (6), 2673-2681

Mok, Y. \& Einaudi, G. 1985 Resistive decay of Alfvén waves in a non-uniform plasma. J. Plasma Phys. 33 (01), 199-208.

Nariyuki, Y., Umeda, T., Suzuki, T. \& Hada, T. 2014a Ion acceleration by parallel propagating nonlinear Alfvén wave packets in a radially expanding plasma. Nonlinear Process. Geophys. 21 (1), 339-346.

NARiYuki, Y., HADA, T. \& Tsubouchi, K. 2014b Collisionless damping of circularly polarized nonlinear Alfvén waves in solar wind plasmas with and without beam protons. Astrophys. J. 793 (2), 138.

Ofman, L., \& Aschwanden, M. 2002 Damping time scaling of coronal loop oscillations deduced from transition region and coronal explorer observations. Astrophys. J. Lett. 576 (2), L153.

Oughton, S., Priest, E. R. \& Matthaeus, W. H. 1994 The influence of a mean magnetic field on three-dimensional magnetohydrodynamic turbulence. J. Fluid Mech. 280, 95-117.

Petkaki, P., Malara, F. \& Veltri, P. 1998 Topological formation of small scales in magnetohydrodynamics: a fast dissipation mechanism. Astrophys. J. $\mathbf{5 0 0}$ (1), 483-491.

Oughton, S., Matthaeus, W. H., Wan, M., \& Osman, K.T. 2015 Anisotropy in solar wind plasma turbulence. Philosophical Transactions of the Royal Society A: Mathematical, Physical and Engineering Sciences 373, 2041, 20140152

Pezzi, O., Parashar, T. N., Servidio, S., Valentini, F., Vásconez, C. L., Yang, Y., Malara, F., Matthaeus, W. H. \& Veltri, P. 2017a Revisiting a classic: the ParkerMoffatt problem. Astrophys. J. 834, 166.

Pezzi, O., Parashar, T. N., Servidio, S., Valentini, F., Vásconez, C. L., Yang, Y., Malara, F., Matthaeus, W. H. \& Veltri, P. 2017b Colliding Alfvénic wave packets in magnetohydrodynamics, Hall and kinetic simulations. J. Plasma Phys. 83, 905830105.

Pezzi, O., Malara, F., Servidio, S., Valentini, F., Parashar, T. N., Matthaeus, W. H. \& VeLtRI, P. 2017c Turbulence generation during the head-on collision of Alfvénic wave packets. Phys. Rev. E 96, 023201.

Pezzi, O., Servidio, S., Perrone, D., Valentini, F., Sorriso-Valvo, L., Greco, A., Matthaeus, W.H., \& Veltri, P. 2018 Velocity-space cascade in magnetized plasmas: numerical simulations. Phys. Plasmas, 25, 060704.

Podesta, J. J. \& TenBarge, J. M. 2012 Scale dependence of the variance anisotropy near the proton gyroradius scale: additional evidence for kinetic Alfvén waves in the solar wind at 1 AU. J. Geophys. Res. 117 (1), A10106.

Pucci, F., Onofri, M. \& Malara, F. 2014 Evolution of magnetohydrodynamic waves in low layers of a coronal hole. Astrophys. J. 796 (1), 43.

Pucci, F., Vásconez, C. L., Pezzi, O., Servisio, S., Valentini, F., Matthaeus, W. H. \& Malara, F. 2016 From Alfvén waves to kinetic Alfvén waves in an inhomogeneous equilibrium structure. J. Geophys. Res. Space Phys. 121, 1024-1045. 
Roberts, D. A., Ghosh, S., Goldstein, M. L. \& Matthaeus, W. H. 1991 MHD simulation of the radial evolution and stream structure of the solar wind turbulence. Phys. Rev. Lett. 67, 3741-3744.

Roberts, D. A., Goldstein, M. L., Matthaeus, W. H. \& Ghosh, S. 1992 Velocity shear generation of solar wind turbulence. J. Geophys. Res. 97, 17,115-17,130.

Roytershteyn, V. \& Daughton, W. 2008 Collisionless instability of thin current sheets in the presence of sheared parallel flows. Phys. Plasmas 15, 082901.

Sahraoui, F., Goldstein, M. L., Robert, P. \& Khotyaintsev, Y. V. 2009 Evidence of a cascade and dissipation of solar-wind turbulence at the electron gyroscale. Phys. Rev. Lett. 102 (23), 231102.

Sahraoui, F., Belmont, G. \& Goldstein, M. 2012 New insight into short-wavelength solar wind fluctuations from Vlasov theory. Astrophys. J. 748 (2), 100.

Salem, C., Howes, G., Sundkvist, D., Bale, S., Chaston, C., Chen, C. \& Mozer, F. 2012 Identification of kinetic Alfvén wave turbulence in the solar wind. Astrophys. J. Lett. 745 (1), L9.

Servidio, S., Chasapis, A., Matthaeus, W.H., Perroned, D., Valentini, F., Parashar, T.N., et al. 2017 Magnetospheric multiscale observation of plasma velocity-space cascade: Hermite representation and theory. Phys. Rev. Lett. 119, 205101.

Schekochinin, A., Cowley, S., Dorland, W., Hammett, G. Howes, G., Quataert, E. \& Tatsuno, T. 2009 Astrophysical gyrokinetics: Kinetic and fluid turbulent cascades in magnetized weakly collisional plasmas. Astrophys. J. Suppl. Ser. 182 (1), 310-377.

Shebalin, J. V., Matthaeus, W. H. \& Montgomery, D. 1983 Anisotropy in MHD turbulence due to a mean magnetic field. J. Plasma Phys. 29 (03), 525-547.

Similon, P. L. \& Sudan, R. 1989 Energy dissipation of Alfvén wave packets deformed by irregular magnetic fields in solar-coronal arches. Astrophys. J. 336, 442-453.

Sorriso-Valvo, L., Perrone, D., Pezzi, O., Valentini, F., Servidio, S., Zouganelis, i. \& VelTRI, P. 2018 Local energy transfer rate and kinetic processes: the fate of turbulent energy in two-dimensional hybrid Vlasov-Maxwell numerical simulations. J. Plasma Phys. 84 (2), 725840201.

Sorriso-Valvo, L., Catapano, F., Retinó, A, Le Contel, O., Perrone, D., Roberts, O.W. et al. 2019 Turbulence-Driven Ion Beams in the Magnetospheric Kelvin-Helmholtz Instability Phys. Rev. Lett. 122, 035102.

Steinolfson, R. 1985 Resistive wave dissipation on magnetic inhomogeneities normal modes and phase mixing. Astrophys. J. 295, 213-219.

TenBarge, J., \& Howes, G. 2012 Evidence of critical balance in kinetic Alfvén wave turbulence simulations. Phys. Plasmas 19 (5), 55901.

Tomczyk, S., McIntosh, S. W., Keil, S., Judge, P., Schad, T., Seeley, D. \& Edmondson, J. 2007 Alfvén waves in the solar corona. Science 317 (5842), 1192-1196.

Tomczyk, S. \& McIntosh S. W. 2009 Time-distance seismology of the solar corona with coMP. Astrophys. J. 697 (2), 1384-1391.

Tsiklauri, D., NAKariakov, V. \& Rowlands, G. 2002 A three dimensional magnetohydrodynamic pulse in a transversely inhomogeneous medium. Astron. Astrophys. 393 (1), 321-329

Tsiklauri, D. \& NAKARIAKov, V. 2003 Phase mixing of a three dimensional magnetohydrodynamic pulse. Astron. Astrophys. 400 (3), 1051-1055.

Tsiklauri, D., Sakai, J.-I. \& SAito, S. 2005 Particle-in-cell simulations of circularly polarised Alfvén wave phase mixing: A new mechanism for electron acceleration in collisionless plasmas. Astron. Astrophys. 435 (3), 1105-1113.

TsiklauRI, D. 2011 Particle acceleration by circularly and elliptically polarised dispersive Alfvén waves in a transversely inhomogeneous plasma in the inertial and kinetic regimes. Phys. Plasmas 18 (9), 92903.

Tsiklauri, D. 2012 Three dimensional particle-in-cell simulation of particle acceleration by circularly polarised inertial Alfvén waves in a transversely inhomogeneous plasma. Phys. Plasmas 19 (8), 82903.

Tu, C.-Y., Marsch, E. \& Qin, Z.-R. 2004 Dependence of the proton beam drift velocity on the proton core plasma beta in the solar wind. J. Geophys. Res. 109, A05101

Valentini, F., Trávníček, P., Califano, F., Hellinger, P. \& Mangeney, A. 2007 
A hybrid-Vlasov model based on the current advance method for the simulation of collisionless magnetized plasma. J. Comp. Phys.. 225, 753.

Valentini, F., Veltri, P., Califano, F. \& Mangeney, A. 2008 Cross-scale effects in solarwind turbulence. Phys. Rev. Lett. 101 (2), 25006.

Valentini, F., Perrone, D. \& Veltri, P. 2011a Short-wavelength electrostatic fluctuations in the solar wind. Astrophys. J. 739 (1), 54

Valentini, F., Califano, F., Perrone, D., Pegoraro, F. \& Veltri, P. 2011b New ion-wave path in the energy cascade. Phys. Rev. Lett. 106 (16), 165002

Valentini, F., Vásconez, C. L., Pezzi, O., Servidio, S., Malara, F. \& Pucci, F. 2017 Transition to kinetic turbulence at proton scales driven by large-amplitude kinetic Alfvén fluctuations. Astron. Astrophys. 599, A8

VÁsconez, C. L., Valentini, F., Camporeale, E. \& Veltri, P. 2014 Vlasov simulations of kinetic Alfvén waves at proton kinetic scales. Phys. Plasmas 21 (11), 112107.

Vásconez, C. L., Pucci, F., Valentini, F., Servidio, S., Matthaeus, W. H. \& Malara, F. 2015 Kinetic Alfvén wave generation by large-scale phase mixing. Astrophys. J. 815, 7

Voitenko, Y., \& Goossens, M. 2004 Cross-field heating of coronal ions by low-frequency kinetic Alfvén waves. Astrophys. J. Lett. 605 (2), L149

Wu, D., \& Chen, L. 2013 Excitation of kinetic Alfvén waves by density striation in magnetoplasmas. Astrophys. J. $\mathbf{7 7 1}$ (1), 3. 